Margaret M. Kuder MD, MPH Department of Allergy and Clinical Immunology,

Respiratory Institute, Cleveland Clinic, Cleveland $\mathrm{OH}$
David M. Lang, MD

Department of Allergy and Clinical Immunology,

Respiratory Institute, Cleveland Clinic, Cleveland $\mathrm{OH}$
Deepa D. Patadia, MD

Department of Allergy and Clinical Immunology,

Respiratory Institute, Cleveland Clinic, Cleveland $\mathrm{OH}$

\title{
Anaphylaxis to vaccinations: A review of the literature and evaluation of the COVID-19 mRNA vaccinations
}

\section{Published February 25, 2021}

\section{ABSTRACT}

Recent reports of allergic reactions to the Pfizer-BioNTech and the Moderna COVID-19 vaccines have resulted in questions about how and to whom they can be safely administered. Although anaphylaxis was not observed in clinical trials for either vaccine, there have been 21 reported possible cases of anaphylaxis associated with the Pfizer vaccine (11.1 cases per million doses administered) and 10 possible cases associated with the Moderna vaccine (2.5 anaphylaxis cases per million doses administered). The etiology of anaphylaxis in these cases is not fully understood and is an area of active research. The overall incidence of anaphylaxis to COVID-19 mRNA vaccines is very low. By following recommendations from the US Centers for Disease Control and Prevention, an overwhelming majority of the US population can be safely immunized.

\section{INTRODUCTION}

Vaccinations against infectious diseases are one of the most impactful public health measures in existence. The development of a vaccine against SARS$\mathrm{CoV}-2$ has been viewed as a tool to control the global COVID-19 pandemic and as a path toward recovery for our society. However, recent reports of allergic reactions to the COVID-19 vaccine have resulted in questions about how and to whom the vaccine can be safely administered. Here we provide a general discussion of vaccine-related reactions. We also discuss current knowledge on COVID-19 vaccine-related

The statements and opinions expressed in COVID-19 Curbside Consults are based on experience and the available literature as of the date posted. While we try to regularly update this content, any offered recommendations cannot be substituted for the clinical judgment of clinicians caring for individual patients.

doi:10.3949/ccjm.88a.ccc075 reactions and recommendations on COVID-19 vaccine administration with respect to potential for allergic reactions.

\section{VACCINE-RELATED ADVERSE REACTIONS}

Adverse reactions to vaccines are commonly reported, but most are not immunologically mediated. Nonimmunologically mediated reactions are typically related to the pharmacology of the vaccine and consist of toxic reactions, side effects, and medication interactions. ${ }^{1}$ Immunologically mediated reactions include IgE-mediated and T-cell mediated reactions as well as other immunologic mechanisms that occur as a result of allergen exposure. These responses, particularly severe anaphylaxis, rarely occur. In the United States (US), anaphylaxis to vaccination is reported at an incidence of 0.7-1.3 per one million vaccinations, ${ }^{2,3}$ and are more common in atopic individuals. ${ }^{3}$ Nevertheless, those with no history of allergic disease may also experience severe adverse reactions. ${ }^{4}$

Most acute-onset immunologically mediated reactions are IgE-mediated, meaning the patient has pre-formed IgE antibodies to a component of the vaccine. In this type of reaction, symptoms can begin within minutes of exposure to the allergen-generally within 30 minutes-and less commonly within several hours. ${ }^{5}$ Symptoms range from urticaria, swelling, and gastrointestinal upset, to respiratory distress and cardiovascular collapse associated with severe anaphylaxis. ${ }^{6}$ Delayed reactions typically occur hours to days after exposure to the antigen trigger, and most typically manifest as a cutaneous reaction. There are reports of delayed-onset reactions to rabies and Japanese encephalitis vaccines, although this is also rare. ${ }^{6}$

Vaccines consist of antigens that elicit the desired immune response, and a variety of other components including adjuvants, carrier proteins, and preservatives. Most IgE-mediated reactions do not occur in 
response to the antigenic components. More commonly, other components are the culprit for allergic reactions. ${ }^{6}$

Often, food components of vaccines have been implicated in vaccine reactions. For instance, patients with anaphylaxis to the MMR vaccine had evidence of IgE-mediated hypersensitivity to gelatin, which is commonly used as a vaccine stabilizing agent. ${ }^{6}$ Milk and egg are common food allergens that are also found in vaccines. Some vaccines are cultured on chicken embryos. However, this typically results in such a small amount of possible egg exposure that even those with severe egg allergy can safely tolerate these vaccines. ${ }^{7}$ Some vaccines employ milk protein as a stabilizer; however, reports of adverse reaction in milk-allergic children are exceedingly rare. ${ }^{6}$

Adjuvants are vaccine components that improve the patient's immune response to the antigen. The most common of these are aluminum hydroxide and aluminum phosphate. These more commonly cause injection site irritation, and there has not yet been a documented case of IgE-mediated hypersensitivity to these ingredients. However, other adjuvants have been implicated. There were reports in Canada of anaphylaxis occurring due to adjuvant system 03 (AS03), an adjuvant used in some influenza vaccines outside of the US. ${ }^{6}$

Latex exposure is another possible culprit of vaccine anaphylaxis. Rubber stoppers or plungers in some vaccine vials contain latex, exposing the patient to trace amounts of latex protein. There are reports of anaphylaxis associated with vaccine-mediated latex exposure. ${ }^{8}$ However, this is rare Individuals with latex allergy should avoid vaccines packaged or administered with latex-containing products. ${ }^{6}$

Preservatives are an anti-microbial component of vaccines that are associated with adverse reactions. However, these are typically limited to local injection site swelling or delayed-hypersensitivity skin reactions. These reactions are rare and are primarily described in single case reports. Thus, even a history of delayed-hypersensitivity reaction to a preservative component, such as thimerosal, is not a contraindication to receiving a thimerosal-containing vaccine. ${ }^{6}$

\section{COVID-19 MRNA VACCINES}

There are currently two COVID-19 vaccines that have been granted emergency use authorization by the FDA: the Pfizer-BioNTech COVID-19 vaccine and the Moderna COVID-19 vaccine. Anaphylaxis was not observed in the clinical trials for either vaccine. Outside of trials, there have been reports in the
US and abroad of anaphylactic reactions occurring after COVID-19 vaccine administration. Based on US data of 1.8 million doses of the Pfizer vaccine administered, there were 175 cases of severe allergic reaction reported to the Vaccine Adverse Events Reporting System (VAERS). Twenty-one of these were deemed consistent with anaphylaxis. Of these, 17 cases occurred in people with a history of allergy or allergic reactions, and $70 \%$ of the reactions occurred within 15 minutes after vaccine administration. ${ }^{9}$ There are 10 reported cases of possible anaphylaxis to the Moderna vaccine (2.5 anaphylaxis cases per million doses administered). ${ }^{10}$ Notably, the PfizerBioNTech has a rate of anaphylaxis four times that of the Moderna vaccine. However, the vaccine components do not differ significantly and the reason for this observed increased reaction rate is unknown. This rate of anaphylaxis is significantly higher than the previously reported one per million vaccination doses seen with other vaccines, but is still quite rare (11.1 cases per million vaccine doses of the Pfizer vaccine) ${ }^{9}$

The etiology of anaphylaxis in these cases is not fully understood and is an area of active research. Any component of a vaccine has the potential to cause adverse reaction. Both COVID-19 vaccines contain mRNA, lipids, polyethylene glycol (PEG)2000, electrolytes, sugar (sucrose), and a saline diluent. Neither contain food products or preservatives. There is no latex exposure because the vial stoppers are not made with natural rubber.

Notably, PEG has been associated with cases of anaphylaxis to other agents. Polyethylene glycol and related polysorbates are ingredients in colonoscopy preps, medications for constipation, vascular grafts, surgical gels, PEGylated medications, and household and industrial compounds and are used as excipients in oral and injectable medications, including vaccines. These components have previously been implicated in anaphylactic reactions. ${ }^{11}$ Currently, patients who exhibit signs of allergic reaction after COVID-19 vaccination are recommended to avoid new PEG exposure and to undergo further evaluation with a board-certified allergist/immunologist.

Another theoretical consideration is complement activation mediated by RNA exposure. In 2008 , reports of adverse reactions to heparin were linked to exposure to oversulfated chondroitin sulfate (OSCS). This was found to induce kallikrein activity and activate the contact system that resulted in anaphylaxis-like symptoms. ${ }^{12}$ It is possible that a nonIgE mediated mechanism could be implicated such as 
TABLE 1

Current CDC recommendations on COVID-19 vaccination

\begin{tabular}{|c|c|c|}
\hline & Clinical history & Action recommended \\
\hline Contraindications & $\begin{array}{l}\text { History of immediate allergic reaction of any severity (in- } \\
\text { cluding anaphylaxis) to mRNA COVID-19 vaccine or any } \\
\text { component of the vaccine (including polyethylene glycol) } \\
\text { History of immediate allergic reaction of any severity to } \\
\text { polysorbate }\end{array}$ & $\begin{array}{l}\text { Vaccine should not be administered } \\
\text { - Refer to allergist/immunologist for further evalu- } \\
\text { ation and to determine if the patient can safely } \\
\text { receive the vaccine }\end{array}$ \\
\hline $\begin{array}{l}\text { No Contraindications } \\
\text { or precautions }\end{array}$ & $\begin{array}{l}\text { History of allergic reactions unrelated to vaccines or } \\
\text { injectable therapy, COVID-19 vaccine components includ- } \\
\text { ing polyethylene glycol, or polysorbate. This includes } \\
\text { those with allergies to foods, latex, gelatin, venom, pets, } \\
\text { environmental allergens, or oral medications }\end{array}$ & $\begin{array}{l}\text { Administer the vaccine } \\
\text { - Patients with history of anaphylaxis from any cause } \\
\text { should be observed for } 30 \text { minutes after administra- } \\
\text { tion } \\
\text { - All other patients should be observed for } 15 \text { min- } \\
\text { utes after administration }\end{array}$ \\
\hline
\end{tabular}

Reprinted from reference 17

complement activation, resulting in anaphylaxis-like symptoms as described with the OSCS.13 Although this is speculative, alternative mechanisms such as this merit further investigation.

To further evaluate possible anaphylaxis associated with the COVID-19 vaccinations, all patients who experience symptoms that are consistent with the clinical criteria for diagnosis of anaphylaxis ${ }^{14,15}$ should have a serum tryptase level and complement components (CH50 and/or SC5b-9) obtained. ${ }^{16}$ These evaluate for mast cell activation seen in anaphylaxis as well as complement system activation. More extensive evaluation, including immediate hypersensitivity skin testing to PEG, can be performed at a later date by a board-certified allergist/immunologist. Patients who have had anaphylaxis with the first dose of the COVID-19 vaccine should be excluded from receiving the second dose.

At this time, there are no evidence-based recommendations for less severe reactions. In our practice, mild, self-limited symptoms (e.g. pruritus or urticaria) are not viewed as absolute contraindications for receiving subsequent dosing of the COVID-19 vaccines. Such patients are given the opportunity to voice their values and preferences via a shared decision making approach, and they may elect to cautiously proceed with receiving the second vaccine dose with antihistamine pre-treatment. These symptoms can be treated symptomatically with oral antihistamines. Allergy and immunology referral for further evaluation may be considered for these patients. Delayed rashes or delayed local reactions (onset $>24$ hours after injection) are unlikely to represent an IgE-mediated allergy, and these individuals may receive the second COVID-19 vaccine dose with no precautions.

With limited data, the recommendations regarding who should and should not receive the vaccine will likely continue to evolve. Currently, the US Centers for Disease Control and Prevention (CDC) has made recommendations regarding the allergic potential of COVID-19 vaccination as outlined in Table $1 .{ }^{17}$

The CDC further recommends that vaccination sites have equipment, supplies, and personnel 
immediately available to manage anaphylaxis. For patients receiving a COVID-19 vaccine, the CDC recommends that those with any history of anaphylaxis should be observed for 30 minutes following vaccination. All other patients may be observed for 15 minutes. $^{18}$

\section{CONCLUSION}

With the administration of increased numbers of the COVID-19 vaccines, cases of anaphylaxis have been reported. The mechanism of these reactions has not yet been fully elucidated. Further investigation should clarify this as we move forward with the vaccine rollout in 2021. Immunization is an important strategy for controlling the COVID-19 pandemic. By following CDC recommendations, an overwhelming majority of the US population can be safely immunized.

\section{DISCLOSURES}

The authors report no relevant financial relationships which, in the context of their contributions, could be perceived as a potential conflict of interest.

\section{REFERENCES}

1. Joint Task Force on Practice Parameters; American Academy of Allergy, Asthma and Immunology; American College of Allergy, Asthma and Immunology; Joint Council of Allergy, Asthma and Immunology. Drug allergy: an updated practice parameter. Ann Allergy Asthma Immunol 2010; 105(4):259-273. doi: 10.1016/j. anai.2010.08.002

2. Bohlke K, Davis RL, Marcy SM, Braun MM, DeStefano F, Black SB, Mullooly JP, Thompson RS; Vaccine Safety Datalink Team. Risk of anaphylaxis after vaccination of children and adolescents. Pediatrics 2003; 112(4):815-820. doi: 10.1542/peds.112.4.815

3. McNeil MM, Weintraub ES, Duffy J, Sukumaran L, Jacobsen SJ, Klein NP, Hambidge SJ, Lee GM, Jackson LA, Irving SA, King JP, Kharbanda EO, Bednarczyk RA, DeStefano F. Risk of anaphylaxis after vaccination in children and adults. J Allergy Clin Immunol 2016; 137(3):868-878. doi: 10.1016/j.jaci.2015.07.048

4. Su JR, Moro PL, Ng CS, Lewis PW, Said MA, Cano MV. Anaphylaxis after vaccination reported to the Vaccine Adverse Event Reporting System, 1990-2016. J Allergy Clin Immunol 2019; 143(4):1465-1473. doi: 10.1016/j.jaci.2018.12.1003

5. Demoly P, Adkinson NF, Brockow K et al. International Consensus on drug allergy. Allergy 2014; 69(4):420-437. doi: 10.1111/all.12350

6. McNeil MM, DeStefano F. Vaccine-associated hypersensitivity. J Allergy Clin Immunol 2018; 141(2):463-472. doi: 10.1016/j. jaci.2017.12.971

7. Greenhawt M, Turner PJ, Kelso JM. Administration of influenza vaccines to egg allergic recipients: A practice parameter update 2017. Ann Allergy Asthma Immunol 2018; 120(1):49-52. doi: 10.1016/j. anai.2017.10.020

8. Russell M, Pool V, Kelso JM, Tomazic-Jezic VJ. Vaccination of persons allergic to latex: a review of safety data in the Vaccine Adverse Event Reporting System (VAERS). Vaccine 2004; 23(5):664-667. doi: 10.1016/j.vaccine.2004.06.042

9. Allergic Reactions Including Anaphylaxis After Receipt of the First Dose of Pfizer-BioNTech COVID-19 Vaccine - United States, December 14-23, 2020. MMWR Morb Mortal Wkly Rep 2021; 70:4651. DOI: http://dx.doi.org/10.15585/mmwr.mm7002e1external icon

10. Allergic Reactions Including Anaphylaxis After Receipt of the First Dose of Moderna COVID-19 Vaccine - United States, December
21, 2020-January 10, 2021. MMWR Morb Mortal Wkly Rep 2021; 70:125-129. DOI: http://dx.doi.org/10.15585/mmwr.mm7004e1external icon

11. Stone CA Jr, Liu Y, Relling MV, et al. Immediate Hypersensitivity to Polyethylene Glycols and Polysorbates: More Common Than We Have Recognized. J Allergy Clin Immunol Pract 2019; 7(5):15331540.e8. doi: 10.1016/j.jaip.2018.12.003

12. Kishimoto TK, Viswanathan K, Ganguly T, et al. Contaminated heparin associated with adverse clinical events and activation of the contact system. N Engl J Med 2008; 358(23):2457-2467. doi: 10.1056/NEJMoa0803200

13. Bender L, Weidmann H, Rose-John S, Renné T, Long AT. Factor XIIDriven Inflammatory Reactions with Implications for Anaphylaxis. Front Immunol 2017; 8:1115. doi: 10.3389/fimmu.2017.01115

14. Sampson HA, Muñoz-Furlong A, Campbell RL, et al. Second symposium on the definition and management of anaphylaxis: summary report-Second National Institute of Allergy and Infectious Disease/ Food Allergy and Anaphylaxis Network symposium. J Allergy Clin Immunol 2006; 117(2):391-397. doi: 10.1016/j.jaci.2005.12.1303

15. Lieberman PL. Recognition and first-line treatment of anaphylaxis. Am J Med 2014; 127(1 Suppl):S6-11. doi: 10.1016/j. amjmed.2013.09.008

16. US Centers for Disease Control and Prevention. Lab Tests to Collect Shortly After Severe Allergic Reaction/Anaphylaxis Following COVID-19 Vaccination. Accessed February 15, 2021. https://www. cdc.gov/vaccines/covid-19/clinical-considerations/anaphylaxis-management.html

17. US Centers for Disease Control and Prevention. Interim Clinical Considerations for Use of mRNA COVID-19 Vaccines Currently Authorized in the United States. https://www.cdc.gov/vaccines/ covid-19/info-by-product/clinical-considerations.html Updated February 10, 2021. Accessed February 24, 2021.

18. US Centers for Disease Control and Prevention. Interim considerations: Preparing for the potential management of anaphylaxis after COVID-19 vaccination. Accessed February 15, 20201. https:// www.cdc.gov/vaccines/covid-19/info-by-product/pfizer/anaphylaxismanagement.html

Correspondence: Margaret M. Kuder, MD, MPH, Department of Allergy and Clinical Immunology, IN8, Cleveland Clinic Independence Family Health Center, 5001 Rockside Road, Independence, OH 44131; kuderm@ccf.org 